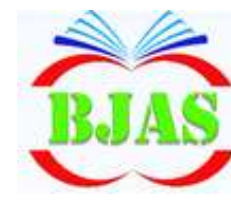

Available online at http://bjas.bajas.edu.iq

https://doi.org/10.37077/25200860.2021.34.2.16

College of Agriculture, University of Basrah

ISSN $1814-5868$

Basrah J. Agric. Sci., 34(2), 204-219, 2021

Basrah Journal

of Agricultural

Sciences

E-ISSN: 2520-0860

\title{
Study of the Antioxidant Activity of Cactus (Opuntia dellienii) Fruits (Pulp and Peels) and Characterisation of their Bioactive Compounds by GC-MS
}

\author{
Alya J. A. Alsaad*\& Lina S. Mohammed ${ }^{1}$ \\ Department of Food Science, College of Agriculture, University of Basrah, Iraq \\ *Correspondence E-mail: alyaalsaad63@yahoo.com: ${ }_{2}^{1}$ linasamer234@gmail.com
}

Received 10 October 2020; Accepted 26 July 2021; Available online 4 November 2021

\begin{abstract}
The present study aims to identify and characterize the chemical compounds present in the extracts of cactus pear Opuntia dillenii (OD) fruit which grow in southern Iraq and offer different health benefits. The physicochemical properties of the fruit juices (pulp and peels) were investigated. Fruit pulp and peel extracts carried the symbols: hexane pulp extract $(\mathrm{HPuE})$, hexane peel extract $(\mathrm{HPeE})$, ethanolic pulp extract (EPuE), and ethanolic peels extract (EPeE). The activel compound screened by gas chromatography-mass spectrometry (GC-MS). HPuE and HPeE contained 40 bioactive compounds and 60 bioactive compounds were detected in $\mathrm{EPuE}$ and $\mathrm{EPeE}$. The scavenging activity of $\mathrm{DPPH}$ free radicals records a high level of inhibition ranging from 34.8 to $90.3 \%$ for $\mathrm{EPeE}$ and from 32.9 to $89.9 \%$ for $\mathrm{EPuE}$, while hexane extracts scavenging activities (HPeE and $\mathrm{HPuE}$ ) were $24.9-78.9 \%$ and 20.7-73.3\% respectively at concentrations of $20-80 \mu \mathrm{g} \cdot \mathrm{ml}^{-1}$ this activity has received considerable attention because of their physiological functions.
\end{abstract}

Keywords: Cactus, DPPH assay, Fruits, GC-MS, Physcochemical parameter.

\section{Introduction}

The southern part of Iraq is distinguished by the abundance of cactus fruits Opuntia dillenii (OD) grown at the Peninsula called Al-Faw. The Opuntia genus belongs to the Cactaceae family, and the common name is prickly pear (Habibi et al., 2002). Arid and semi-arid regions are special areas for cultivating cactus fruits that are geographically distributed in Mexico, Latin America, South Africa, and Mediterranean countries (Butera et al., 2002). O. dillenii plants have a high environmental adaptivity and adapt to all climatic conditions due to their remarkable genetic (Moßhammer et al., 2006). Opuntia fruit is a berry with a delicately flavored juicy pulp that contains many hard seeds and has a thick pericarp (peel) of $30-40 \%$ of the fruit (Duru \&Turker, 2005). Cactus pear acidity is $0.03-0.12 \%$, while the $\mathrm{pH}$ range from 6.0 to 6.6 , and total soluble solids was $12-17 \%$ (Sharma et al., 2015). Fruit peels contain high amounts of flavonoids, polyphenols, betacyanins, and dyes, offering several health benefits (Koubaa et al., 2015). Current studies have shown that pear fruit contains many special chemical components that provide this fruit multiple functionalities and increased nutritional value, attracting scientist's research interest in recent years (Piga, 2004). Cactus pear fruit 
and peel extracts have important properties, such as anticancer and, anti-inflammatory effects (Madrigal-Santillán et al., 2013). Cactus pear fruits contain vitamin E, ascorbic acid, amino acids, carotenoids, and fibres enriched in carbohydrates like fructose and glucose, which provide the body strength and health (Valente et al., 2010; Osorio-Esquivel et al., 2011). Cactus peels contain liposoluble compounds and essential fatty acids used as antioxidants (Ramadan \& Mörsel, 2003). Antioxidants could inhibit the oxidation of lipid and cactus extracts utilise as natural food additives for anti-rancidity (Alsaad et al., 2019). Antioxidants compounds, such as phenolic compounds, could delay oxidative effects in DNA, lipids, and proteins by producing astable radicals (Shahidi et al., 1992). Some chemically synthesised antioxidants, such as Butylated Hydroxytoluene (BHT) and Butylated Hydroxyanisole (BHA) have toxic effects (Zhang et al., 2009); therefore, natural antioxidants from plant resources are of great interest among researchers (Buyukokuroglu et al., 2001; Gulcin et al., 2003). To our information, this study is the first study in Iraq that analyses the active compounds and other properties of prickly pear. Therefore, the objectives of this study are to (i) identify the bioactive compounds of cactus (OD) fruit (pulp and peels) extracts by using GC-MS to describe the nutraceutical compounds which are useful for human health and (ii) quantify their physicochemical properties and antioxidant activity.

\section{Materials \& Methods}

\section{Materials and reagents}

Butylated Hydroxytoluene (BHT), 2,2diphenyl-1picrylhydrazyl (DPPH), a doublebeam UV-VIS spectrophotometer to measure intensity of light beam. All solvents used for partition and extraction were purchased from Sigma-Aldrich (St.Louis, MO, USA).

\section{Plant materials}

Cactus fruit Opuntia dellienii (OD) grows in abundance in the southern part of Iraq, Basrah. The temperature ranges between $48^{\circ} \mathrm{F}$ and $108^{\circ} \mathrm{F}$ with small amounts of rain during the year. Sandy and silt soils are predominant. Fruits were harvested at their full maturity in August 2019. Only fresh, clean, free of defects, uniform-sized, good-textured fruits were procured for the experiments. One local genotype (purple) was selected for the test. Fifty fruits were used for the experiments. Seeds were manually separated from the pulp and the peels. Then, the fruit pulp and peels were briefly homogenised in a kitchen-type blender. The juice was centrifuged at $(4000 \mathrm{x}$ g, $30 \mathrm{~min}$ at $4^{\circ} \mathrm{C}$ ), and then the supernatant juice was immediately used to measure physicochemical parameters.

\section{Physicochemical parameters}

Based on the procedures described in AOAC (2010), the moisture of fresh Cactus pulp and peels and total sugars of their juices were determined. The total soluble solids (TSS) ( ${ }^{\circ}$ Brix) was determined using a refractometer (DBR95, G. Bormac, Carpi, Italy). The pH was measured using a pH meter (Model 430, Corning, NY, USA). Total acidity was measured by mixing $10 \mathrm{ml}$ of juice with $0.1 \mathrm{~N}$ $\mathrm{NaOH}$ solution until reaching a $\mathrm{pH} 8.1$, which was observed potentiometrically. The results were expressed as \% citric acid (Matias et al., 2014). All parameters were carried out in triplicates.

\section{Preparation of extracts}

$100 \mathrm{~g}$ of cactus (OD) fruit pulp and peels without seeds were dried at $45-50{ }^{\circ} \mathrm{C}$ for 48 
$\mathrm{h}$, ground in an electric grinder to transform it into powder form. Polar and nonpolar compounds were extracted by adding $150 \mathrm{ml}$ of ethanol and hexane solution to $5 \mathrm{~g}$ of each pulp and peel powders and leaving the solution soaked for two days. Filter paper No1 was used to filter extract solutions. The extract was then concentrated using a rotary evaporator at $45{ }^{\circ} \mathrm{C}$, and dry extracts were obtained, Fig. (1). The extracts were kept in a glass container away from light and stored at $4{ }^{\circ} \mathrm{C}$ until further use. The extracts carried the symbols: hexane pulp extract (HPuE), hexane peels extract (HPeE), ethanolic pulp extract $(\mathrm{EPuE})$, and ethanolic peels extract (EPeE). All analyses were carried out in triplicates. Extracts yield was determined on a dry basis (DW) according to the following equation:

$$
\% \text { Yield }=\frac{\mathrm{W} 1 \times 100}{\mathrm{~W} 2}
$$

where $\mathrm{W} 1$ =extract weight after evaporation of the solvent and $\mathrm{W} 2=$ weight of dried fruits.
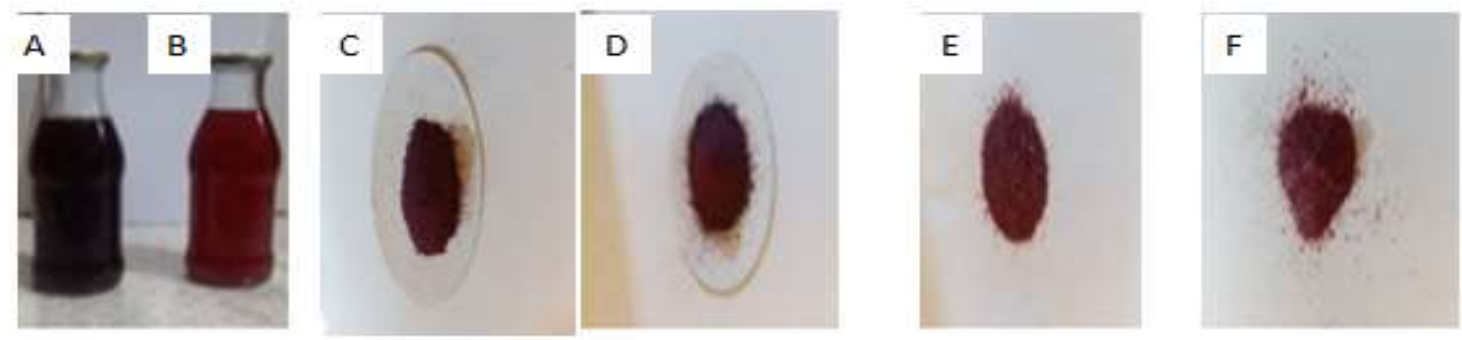

Fig.(1): Iraqi cactus Opuntia dellienii (OD) fruit extracts studied in this research, pulp juice $(A)$, peels juice (B), ethanolic peels extract $(C)$, hexane peels extract $(D)$, ethanolic pulp extract (E), hexane pulp extract $(\mathrm{F})$.

\section{Identification of organic compounds}

The organic compounds of Cactus extracts were analysed using Gas ChromatographyMass Spectrometer (GC-MS) technique (Shimadzu QP2010). A sample of 1 microliter was placed into the capillary column (intercut DB5MS, Japan). The initial column temperature was $50{ }^{\circ} \mathrm{C}$ which rising at the rate of $5 \mathrm{C}^{\circ}$ per min to a final temperature of 250 ${ }^{\circ} \mathrm{C}$. The system provides a carbowax (ID 30 $\mathrm{m} \times 0.25 \mathrm{~mm}$; film thickness $0.25 \mu \mathrm{m}$. As a carrier gas, Helium gas was used. Split mode (1:30) was carried out for injection. The temperatures of the detector and injector were adjusted at $250{ }^{\circ} \mathrm{C}$. Organic compounds were isolated at stable pressure $(90 \mathrm{kPa})$. The identification of chemical compounds in various extracts was based on GC retention time. The mass spectra were computer matched with those of standards available in mass spectrum libraries (2008NIST Library).

\section{Determination of (DPPH) free radical}

The scavenging activity of 1,1-diphenyl-2 picrylhydrazyl (DPPH) of all extracts of cactus (OD) pulp and peels was measured using DPPH assay following the described procedure by Morales \& 'Enez-Perez (2001). Each extract was dissolved in methanol at concentrations of 20, 40, 60, and $80 \mu \mathrm{g} \cdot \mathrm{ml}^{-1}$. The reaction mixture contained $375 \mu$ solvent (methanol), $125 \mu \mathrm{l}$ of $1 \mathrm{mM}$ of DPPH prepared in methanol, and $500 \mu \mathrm{l}$ of extract. After incubation for $30 \mathrm{~min}$ at $25{ }^{\circ} \mathrm{C}$, when the reaction mixture changed its colour from deep violet to light yellow, the absorbance 
was read at $517 \mathrm{~nm}$. As a comparative

(\%) was determined from the equation:

sample, BHT was used. The inhibition ratio

$$
\% \mathrm{DPPH}=\frac{\text { Abs control }- \text { Abs sample }}{\text { Abs control }} \times 100
$$

Where Abs control is control absorbance, and Abs sample is sample absorbance.

\section{Statistical analysis}

The data of triplicate measurements were expressed as the mean \pm standard deviation (SD). The SPSS version 17.0 software (SPSS Inc., Chicago, IL, USA) was used for statistical analyses of the data. One-way analysis of variance (ANDVA) was used to sanalyse the results. Tukey test for multiple comparisons was conducted to show the differences between the means at $p=0.05$.

\section{Results \& Discussion}

\section{Physicochemical parameters}

The physicochemical parameters results of cactus fruit juices (pulp and peels) are given in table (1). Fresh fruit pulp and peels contain $84.9 \%$ and $71.4 \%$ of water, respectively. The pulp had significantly $(p<0.05)$ higher water content than peels. Each cultivar of the fruit has special physicochemical properties and the environment has a major impact on these properties (Parish \& Felker, 1997). Our results are consistent with Gurrier et al. (2000), who found that cactus fruit pulp contains $85 \%$ water. Anwar \& Sallam (2016) studied prickly pear (Opuutia ficusindica) and found that peels contain $75.8 \%$ water. The fruits with high water content, such as prickly pears, are prone to rapid spoilage at room temperature (Bouzoubaâ et al., 2016). The moisture content of other fruit peels, such as pitaya peels (93\%), carrot $(91.05 \%)$, red apple $(81.68 \%)$, and white grape $(75.28 \%)$ was different from that of cactus peels (Makris et al., 2007).

The values of TSS $\left({ }^{\circ}\right.$ Brix) of cactus fruit juice were 14.30 and 14.20 for pulp and peels, respectively. There was no significant difference $(p>0.05)$ between the TSS of pulp and peels. TSS increased during ripening and varied at different maturity and fruit metabolism stages during ripening (Albano et al., 2015). Our results were in accordance with Bouzoubaâ et al. (2016), who found TSS ( ${ }^{\circ}$ Brix) of prickly pears ranging 13.15 - 15.87 .

The average $\mathrm{pH}$ values obtained for pulp and peels in our study were 5.01 and 5.63, respectively. The $\mathrm{pH}$ of pulp was lower than that of peels. There was a significant difference $(p<0.05)$ between the $\mathrm{pH}$ of pulp and peels. The $\mathrm{pH}$ value of the prickly pear juice was low because of the presence of extremely high content of organic acids, such as acetic acid, citric acid, and nhexadecanoic acid. In contrast, the acidity of the pulp was found to be $0.15 \%$; this value is higher compared to the acids content of the peels $0.06 \%$. It is assumed that these fruits were fully mature and low acidic food. Our results are consistent with the results of Sáenz (2000) who revealed that the acidity of cactus fruit ranged from 0.05 to $0.18 \%$. Gurrieri et al. (2000) found the $\mathrm{pH}$ value of peels ranging from 6.4 to 6.5 with acidity ranging between 0.11-0.14. Other fruit juices acidity values, such as orange $(0.94 \%)$, Green grapes (1.06\%), Apple $(0.48 \%)$ Lemon $(9.65 \%)$ (Yadav \& Chakravarty, 2013) 
Our study found that cactus (OD) fruits contain a high amount of total sugar (39.6, $21.9 \%$ ), alsorepresented by reducing sugar (34.4 and 18.9\%) and non-reducing sugar $(5.2,3.0 \%)$ of fruit pulp and peels juices, respectively. In general, pulps taste sweeter than peels. There is a significant difference $(p<0.05)$ between pulp and peels. Our analysis confirmed these results that pulp contained large amounts of carbohydrate represented by d-glycerol-dgalactoheptose, fructose, and lactose. Usually, the fruits of dry soils are sweeter than wetland soil (Jacobo, 2001). The total sugar of prickly pear peels was $60.58 \%$ (Anwar \& Sallam, 2016). Fructose and glucose were the main sugar content of prickly pear juice (11-12\%) (Gurrieri et al., 2000).

Table (1): Physicochemical parameters of cactus $O$. dillenii (OD) fruit juice.

\begin{tabular}{lccccc}
\hline Samples & Moisture $\%$ & $\operatorname{TSS}^{\circ}($ Brix $)$ & $\mathrm{pH}$ & Acidity\% & $\begin{array}{l}\text { Total } \\
\text { sugar\% }\end{array}$ \\
\hline Pulp juice & $84.9 \pm 0.05^{\mathrm{a}}$ & $14.30 \pm 0.11^{\mathrm{a}}$ & $5.01 \pm 1.0^{\mathrm{a}}$ & $0.15 \pm 1.01^{\mathrm{a}}$ & $39.6 \pm 0.40^{\mathrm{a}}$ \\
\hline Peels juice & $74.40 \pm 0.01^{\mathrm{b}}$ & $14.20 \pm 0.21^{\mathrm{a}}$ & $5.63 \pm 0.1^{\mathrm{b}}$ & $0.06 \pm 0.01^{\mathrm{b}}$ & $21.9 \pm 0.03^{\mathrm{b}}$ \\
\hline
\end{tabular}

- Mean with the different letters in the same column is significantly different $(\mathrm{p} \leq 0.05)$, Each value is the means \pm standard deviation $(n=3)$

\section{Yield}

The yield of extracts obtained from cactus (OD) peels, and pulp was given in table (2). The yield of extracts ranged between1.24.1\%. Among all extracts, EPeE has the highest yield $(4.1 \%)$ followed by $\mathrm{EPuE}$ (3.9\%). The yields of HPeE and HPuE were 1.4 and $1.2 \%$, respectively. There is asignificant $(p>0.05)$ difference in the yield between the ethanolic extracts and hexane extracts. The yield of extraction depends on the type of extraction solvents; hexane produced the lowest volume of extracts, whereas ethanol produced the highest amount of extracts. Our results confirmed that the highest extraction occurred for a high polar solvent. Fernández-López et al. (2010) showed that the yield of ethanolic peels extracts of $O$. ficus indica had 64.745 $\mu \mathrm{g} .100 \mathrm{~g}^{-1}$, methanolic extracts $50.75 \mu \mathrm{g} .100 \mathrm{~g}^{-}$ 1 , while chloroform and acetone extracts were recorded the lowest values, 4.8 and $2.5 \mu \mathrm{g} \cdot 100 \mathrm{~g}^{-1}$. Koubaa et al. (2015) studied two varieties of O.acanthocarpa and found that their extraction yields were $0.05 \%$ and $0.07 \%$.

Table (2): \% yield of cactus $O$. dillenii (OD) fruits extracts.

\begin{tabular}{lll}
\hline Samples & Ethanolic extracts & Hexane extracts \\
\hline Peels & $4.1 \pm 0.01^{\mathrm{aA}}$ & $1.4 \pm 0.04^{\mathrm{bB}}$ \\
\hline Pulp & $3.9 \pm 0.10^{\mathrm{aA}}$ & $1.2 \pm 0.05^{\mathrm{bB}}$ \\
\hline
\end{tabular}


- The same small letters in the same row are not significantly different $(p>0.05)$. The mean with the same capital letters in the same column is not significantly different $(p>0.05)$. Each value is the means \pm standard deviation $(\mathrm{n}=3)$

\section{Organic compounds identification}

GC-MS is an effective technique for finding the composition of volatile components of plant origin (Butera et al., 2002). Figs. (2 and 3) of GC-MS chromatogram of cactus (OD) extracts (pulp and peels) clearly show 20 peaks for each extract of HPuE and HPeE, indicating the presence of 20 compounds of various classes of bioactive chemical constituents. At the same time, each EPuE and EPeE had 30 compounds (Figs. 4 and 5). Tables ( 3 and 6) show the extracts compounds with the retention time (RT), peak area, molecular weight (MW), and formula for HPuE, HPeE, EPuE, and EPeE, respectively. The chemical compositions of the four extracts varied quantitatively and qualitatively. Tables (3 and 4) show that $37.94 \%$ of HPuE compounds were Hexanedioic acid dioctyl ester, Decanoic acid, 2-propenyl ester $9.15 \%$. The compound 1Decanol cover $10.69 \%$, phenolic compounds represented by (Z)-3-(pentadic-8-en-1-yl) phenol with levels containing up to $4.29 \%$. The extract HPeE contains Eicosanoic acid (Arachidic acid) in a percentage of $18.04 \%$, nFatty alcohol: Z, Z-8, 10-Hexadecadien-1-ol with levels containing up to $19.25 \%$ and Pentalene, octahedron-2-[(2-octyl) decyl]$11.15 \%$, phenolic compounds: (Z)-3(pentadic-8-en-1yl) phenol comprised $6.01 \%$. Our results were in agreement with those reported by Zito et al. (2013) who showed that $(14.0 \%)$ of volatile compounds were present in $O$. ficus indica fruits. These results also are agreement with data reported by Bergaoui et al. (2007) who found that the most important compounds in cladodes, flowers, and fruits of Opuntia lindheimeri were saturated fatty acids represented by hexadecanoic acid (8.5$17.3 \%)$, tetradecanoic acid (3.2-13.6\%), and butyl tetradecanoate (8.1-21.5\%), contributing antioxidant and anti-bacterial properties (Rajeswari et al., 2012; Aghajani et al., 2015). Ethanolic pulp and peels extracts (EPuE and EPeE) contained the same compounds (Tables 5 and 6) represented by dGlycerol-d-galactoheptose 29.27 and $19.58 \%$, Butoxyacetic acids 23.59 and $11.39 \%$, 7Tetradecenal,(Z)- 13.54 and 22.08\%, 2Methoxy-4-vinyl phenol 6.11 and $10.84 \%$ respectively. In comparison, citric acid $4.23 \%$ presented in EPeE only. Our results agree with Flath \& Takahashi (1978), who found that $O$. ficus-indica peels contain numerous esters and carbonyl compounds. Jaiswal et al. (2009) isolated polysaccharides derived from Opuntia spp. by using an ultrasonic extraction method and showed that it contained various compounds of sugars represented by arabinose, xylose, fructose, glucose, galacturonic acid, and rhamnose units.

It is evident that the extracts found in this study have different biological compounds, such as phenolic compounds, fatty acid aster and citric acid which acts as chelators and synergists of antioxidant systems (Sarkadei \& Howell, 2007). 
Alsaad \& Mohammed / Basrah J. Agric. Sci., 34(2), 204-219, 2021

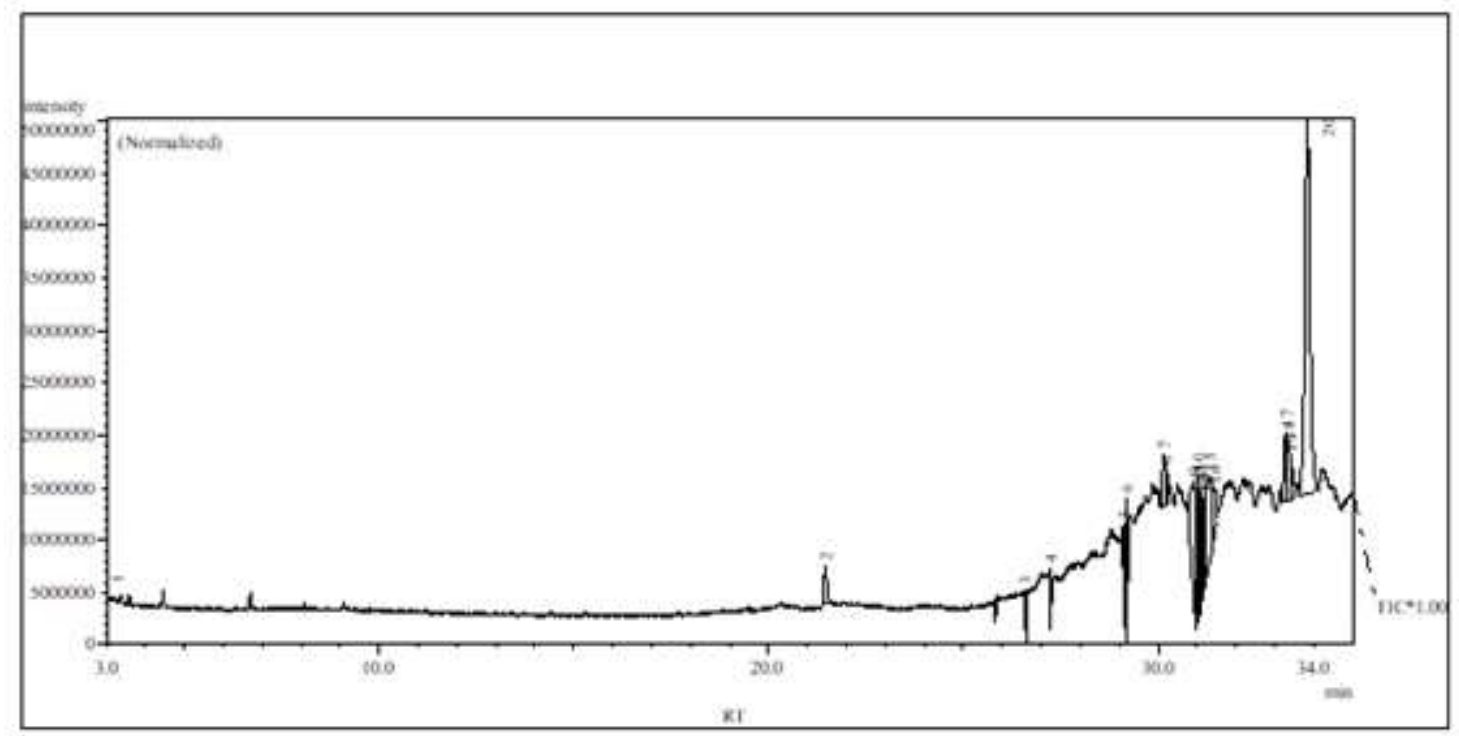

Fig. (2): GC-MS chromatogram of HPuE of cactus $O$. dillenii (OD) fruit.

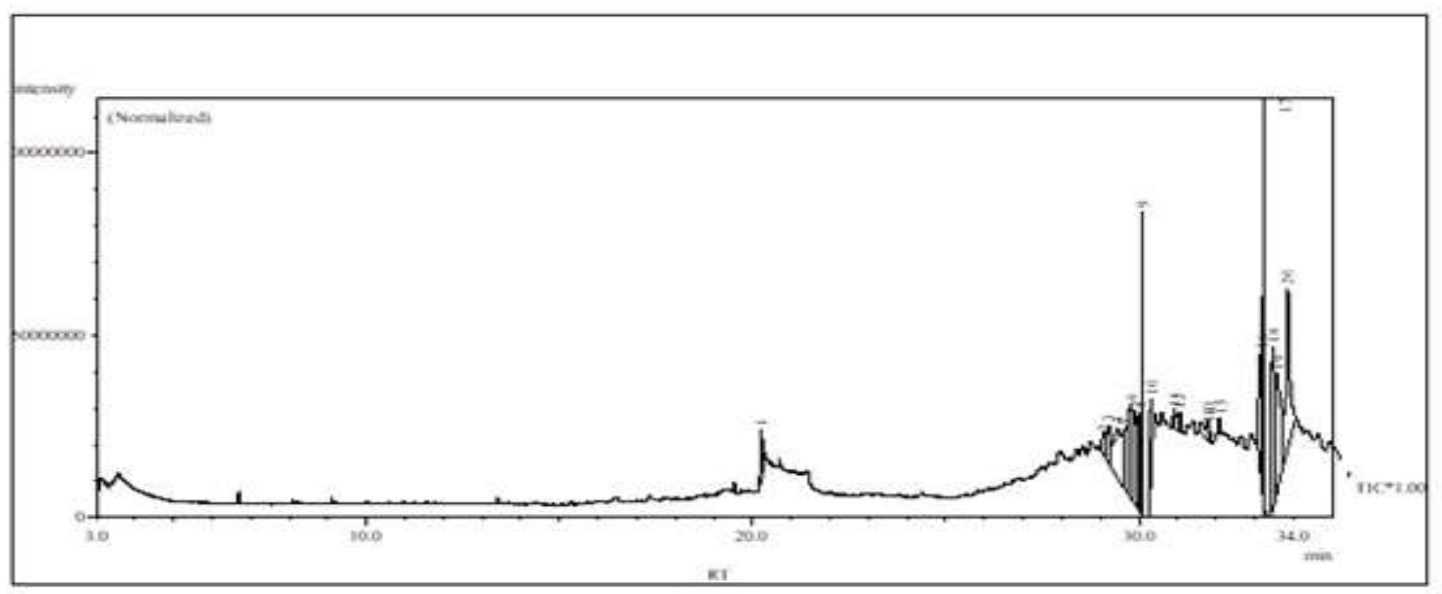

Fig. (3): GC-MS chromatogram of $\mathrm{HPeE}$ of cactus $O$. dillenii (OD) fruit.

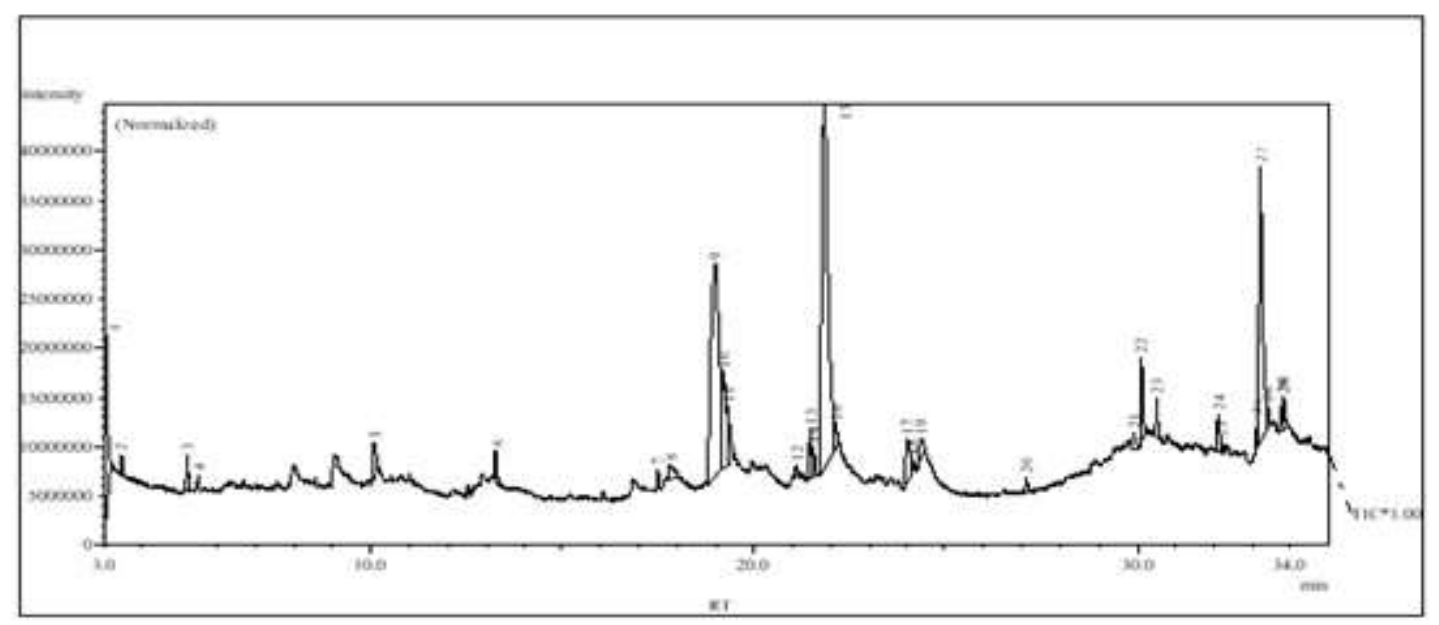

Fig. (4): GC-MS chromatogram of EPuE of cactus $O$. dillenii (OD) fruit. 
Alsaad \& Mohammed / Basrah J. Agric. Sci., 34(2), 204-219, 2021

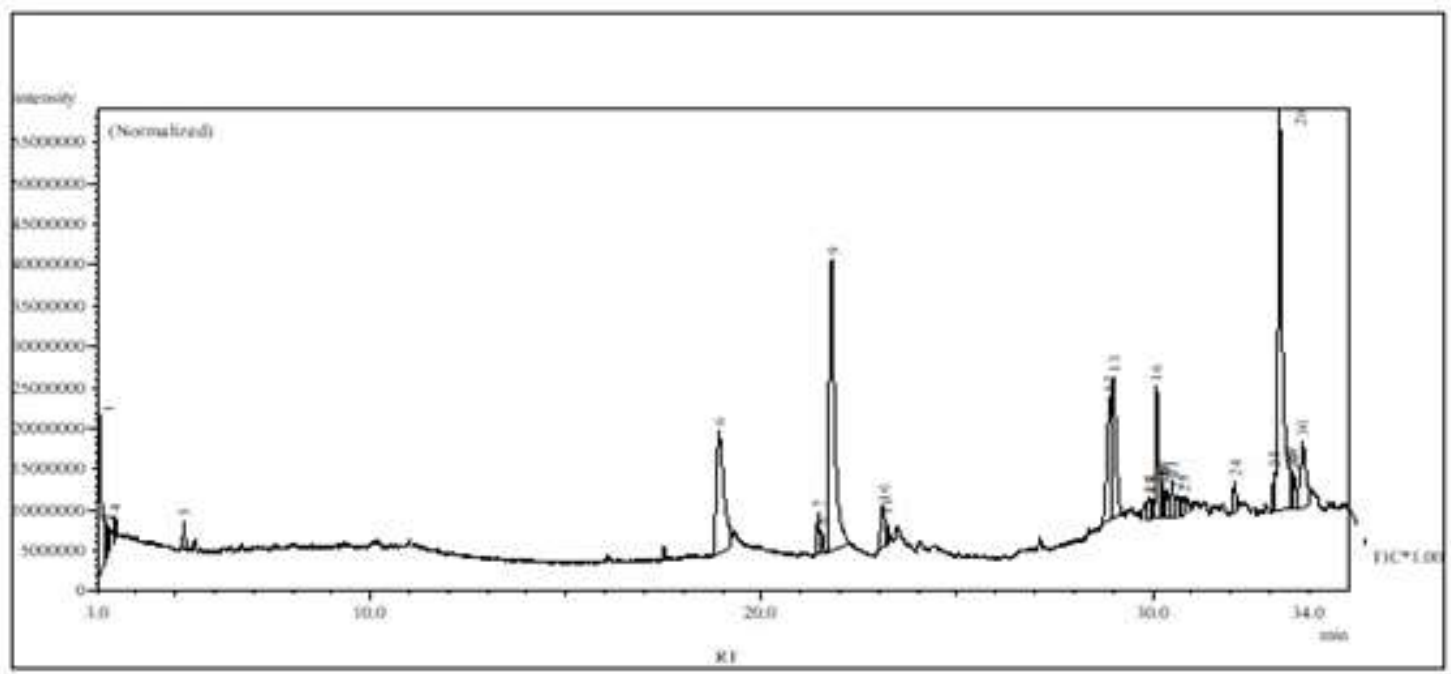

Fig. (5): GC-MS chromatogram of EPeE of cactus $O$. dillenii (OD) fruit.

Table (3): GC-MS analysis of HPuE of cactus $O$. dillenii (OD) fruit.

\begin{tabular}{|c|c|c|c|c|c|}
\hline $\mathrm{Pk}$ & $\mathrm{RT}(\min )$ & $\%$ Area & Formula & MW. & Compounds name \\
\hline 1 & 3.023 & 0.32 & $\mathrm{C} 4 \mathrm{H} 6 \mathrm{~N} 2 \mathrm{O}$ & 98 & 1H-Pyrrole, 2,5-dihydro-1-nitroso- \\
\hline 2 & 21.466 & 2.23 & $\mathrm{C} 12 \mathrm{H} 14 \mathrm{O} 4$ & 222 & Diethyl Phthalate \\
\hline 3 & 26.603 & 0.79 & $\mathrm{C} 22 \mathrm{H} 40 \mathrm{O} 4$ & 368 & Oxalic acid, cyclobutylhexadecyl ester \\
\hline 4 & 27.249 & 0.57 & $\mathrm{C} 21 \mathrm{H} 34 \mathrm{O}$ & 302 & (Z)-3-(pentadec-8-en-1-yl)phenol \\
\hline 5 & 29.144 & 3.72 & $\mathrm{C} 21 \mathrm{H} 34 \mathrm{O}$ & 302 & (Z)-3-(pentadec-8-en-1-yl)phenol \\
\hline 6 & 29.207 & 2.85 & $\mathrm{C} 14 \mathrm{H} 30 \mathrm{O}$ & 214 & 2-Hexyl-1-octanol \\
\hline 7 & 30.151 & 3.27 & $\mathrm{C} 16 \mathrm{H} 32 \mathrm{O} 2$ & 256 & n-Hexadecanoic acid \\
\hline 8 & 30.233 & 0.9 & $\mathrm{C} 35 \mathrm{H} 72 \mathrm{O}$ & 508 & 1-Pentatriacontanol \\
\hline 9 & 30.911 & 10.69 & $\mathrm{C} 16 \mathrm{H} 34 \mathrm{O}$ & 242 & 1-Decanol \\
\hline 10 & 31.028 & 3.47 & $\mathrm{C} 41 \mathrm{H} 84 \mathrm{O}$ & 592 & 1-Hentetracontanol \\
\hline 11 & 31.06 & 2.79 & C7H11NO & 125 & 2-Propionyl-1-pyrroline \\
\hline 12 & 31.087 & 1.53 & $\mathrm{C} 39 \mathrm{H} 76 \mathrm{O} 3$ & 592 & Oleic acid, 3-(octadecyloxy)propyl ester \\
\hline 13 & 31.127 & 3.22 & $\mathrm{C} 19 \mathrm{H} 34 \mathrm{O}$ & 278 & 1,9,12-Octadecatriene, 1-methoxy- \\
\hline 14 & 31.2 & 3.98 & C6H11NO & 113 & N-Allyl-N-ethylformamide \\
\hline 15 & 31.347 & 9.15 & $\mathrm{C} 13 \mathrm{H} 24 \mathrm{O} 2$ & 212 & Decanoic acid, 2-propenyl ester \\
\hline 16 & 31.48 & 3.85 & C26H52 & 364 & 1-Hexacosene \\
\hline 17 & 33.293 & 4.21 & $\mathrm{C} 14 \mathrm{H} 24 \mathrm{O}$ & 208 & 13-Tetradece-11-yn-1-ol \\
\hline
\end{tabular}


Alsaad \& Mohammed / Basrah J. Agric. Sci., 34(2), 204-219, 2021

\begin{tabular}{lrrlll}
\hline $\mathbf{1 8}$ & 33.36 & 2.53 & $10 \mathrm{H} 18 \mathrm{O}$ & 154 & 2-Isopropyl-5-methylhex-2-enal \\
\hline $\mathbf{1 9}$ & 33.413 & 1.99 & $\mathrm{C} 25 \mathrm{H} 50$ & 350 & Heptadecane, 9-(2-cyclohexylethyl)- \\
\hline $\mathbf{2 0}$ & 33.843 & 37.94 & $\mathrm{C} 22 \mathrm{H} 42 \mathrm{O} 4$ & 370 & Hexanedioic acid, dioctyl ester \\
\hline
\end{tabular}

Table (4): GC-MS analysis of HPeE of cactus $O$. dillenii (OD) fruit.

\begin{tabular}{|c|c|c|c|c|c|}
\hline $\mathrm{PK}$ & $\mathrm{RT}(\min )$ & $\%$ Area & Formula & MW & Compounds name \\
\hline $\mathbf{1}$ & 20.228 & 2.16 & $\mathrm{C} 12 \mathrm{H} 14 \mathrm{O} 4$ & 222 & Diethyl Phthalate \\
\hline 2 & 29.06 & 1.49 & $\mathrm{C} 43 \mathrm{H} 88$ & 604 & Tetracontane, 3,5,24-trimethyl- \\
\hline 3 & 29.223 & 2.38 & $\mathrm{C} 40 \mathrm{H} 77 \mathrm{~F} 3 \mathrm{O} 2$ & 646 & Octatriacontyltrifluoroacetate \\
\hline 4 & 29.433 & 8.56 & C38H69F7O2 & 690 & Tetratriacontylheptafluorobutyrate \\
\hline 5 & 29.633 & 4.17 & $\mathrm{C} 26 \mathrm{H} 50$ & 362 & Cyclopentane, 1,1'-hexadecylidenebis- \\
\hline 6 & 29.793 & 6.02 & $\mathrm{C} 20 \mathrm{H} 38 \mathrm{O} 2$ & 310 & cis-11-Eicosenoic acid \\
\hline 7 & 29.9 & 5.44 & $\mathrm{C} 16 \mathrm{H} 22 \mathrm{O} 4$ & 278 & Dibutyl phthalate \\
\hline 8 & 30.007 & 6.48 & C17H36O3S & 320 & Sulfurous acid, dodecyl pentyl ester \\
\hline 9 & 30.094 & 5.83 & $\mathrm{C} 20 \mathrm{H} 40 \mathrm{O} 2$ & 312 & Eicosanoic acid \\
\hline 10 & 30.303 & 3.14 & $\mathrm{C} 34 \mathrm{H} 68 \mathrm{O} 2$ & 508 & Heptadecanoic acid, heptadecyl ester \\
\hline 11 & 30.913 & 1.41 & C29H58 & 406 & $\begin{array}{l}\text { Cyclopentane, 1-(2-decyldodecyl)-2,4- } \\
\text { dimethyl- }\end{array}$ \\
\hline 12 & 31.067 & 0.73 & $\mathrm{C} 21 \mathrm{H} 44$ & 296 & Heptadecane, 2,6,10,15-tetramethyl- \\
\hline 13 & 31.786 & 1.86 & $\mathrm{C} 14 \mathrm{H} 30 \mathrm{O}$ & 214 & 2-Hexyl-1-octanol \\
\hline 14 & 31.827 & 1.26 & $\mathrm{C} 22 \mathrm{H} 37 \mathrm{NO} 4$ & 379 & 2-Nitro-1,3-bis-octyloxy-benzene \\
\hline 15 & 32.078 & 0.46 & $\mathrm{C} 16 \mathrm{H} 32 \mathrm{O}$ & 240 & Hexadecen-1-ol, trans-9- \\
\hline 16 & 33.153 & 6.0 & $\mathrm{C} 21 \mathrm{H} 34 \mathrm{O}$ & 302 & (Z)-3-(pentadec-8-en-1-yl)phenol \\
\hline 17 & 33.245 & 12.03 & $\mathrm{C} 16 \mathrm{H} 30 \mathrm{O}$ & 238 & Z,Z-8,10-Hexadecadien-1-ol \\
\hline 18 & 33.456 & 7.22 & $\mathrm{C} 16 \mathrm{H} 30 \mathrm{O}$ & 238 & Z,Z-8,10-Hexadecadien-1-ol \\
\hline 19 & 33.567 & 11.15 & $\mathrm{C} 26 \mathrm{H} 50$ & 362 & $\begin{array}{l}\text { Pentalene, } \\
\text { octyl)decyl]- }\end{array}$ \\
\hline 20 & 33.861 & 12.21 & $\mathrm{C} 20 \mathrm{H} 40 \mathrm{O} 2$ & 312 & Eicosanoic acid \\
\hline
\end{tabular}


Alsaad \& Mohammed / Basrah J. Agric. Sci., 34(2), 204-219, 2021

Table (5): GC-MS analysis of EPuE of cactus O. dillenii (OD) fruit.

\begin{tabular}{|c|c|c|c|c|c|}
\hline $\mathrm{Pk}$ & $\mathrm{RT}(\min )$ & $\%$ Area & Formula & MW & Compounds name \\
\hline 1 & 3.078 & 5.25 & C7H14O2 & 130 & Acetic acid, 3-methylbutyl ester \\
\hline 2 & 3.471 & 0.34 & $\mathrm{C} 6 \mathrm{H} 14 \mathrm{O}$ & 102 & Propane, 1-(1-methylethoxy)- \\
\hline 3 & 5.232 & 0.78 & $\mathrm{C} 9 \mathrm{H} 20 \mathrm{O} 2$ & 160 & Butane, 1,1-diethoxy-3-methyl- \\
\hline 4 & 5.517 & 0.22 & $\mathrm{C} 8 \mathrm{H} 18 \mathrm{O} 3$ & 162 & Ethyl orthoacetate \\
\hline 5 & 10.077 & 1.63 & $\mathrm{C} 9 \mathrm{H} 10 \mathrm{O} 2$ & 150 & 2-Methoxy-4-vinylphenol \\
\hline 6 & 13.235 & 0.77 & $\mathrm{C} 7 \mathrm{H} 12 \mathrm{O} 3$ & 144 & 4-Pentanoic acid, 2-methoxy-, methyl ester \\
\hline 7 & 17.503 & 0.36 & $\mathrm{C} 14 \mathrm{H} 28$ & 196 & 1-Tetradecene \\
\hline 8 & 17.814 & 1 & $\mathrm{C} 11 \mathrm{H} 24 \mathrm{O} 2$ & 188 & $\begin{array}{l}\text { Butane, } \\
\text { methyl- }\end{array}$ \\
\hline 9 & 18.975 & 23.59 & $\mathrm{C} 6 \mathrm{H} 12 \mathrm{O} 3$ & 132 & Butoxyacetic acid \\
\hline 10 & 19.21 & 4.23 & $\mathrm{C} 6 \mathrm{H} 8 \mathrm{O} 7$ & 192 & Citric Acid \\
\hline 11 & 19.327 & 1.97 & $\mathrm{C} 9 \mathrm{H} 10 \mathrm{O} 2$ & 150 & 2-Methoxy-4-vinylphenol \\
\hline 12 & 21.092 & 0.64 & $\mathrm{C} 11 \mathrm{H} 22 \mathrm{O} 2$ & 186 & Undecanoic acid \\
\hline 13 & 21.457 & 1.39 & $\mathrm{C} 12 \mathrm{H} 14 \mathrm{O} 4$ & 222 & Diethyl Phthalate \\
\hline 14 & 21.541 & 0.8 & $\mathrm{C} 13 \mathrm{H} 28 \mathrm{O}$ & 200 & n-Tridecan-1-ol \\
\hline 15 & 21.806 & 29.72 & C7H14O7 & 210 & d-Glycero-d-galactoheptose \\
\hline 16 & 22.127 & 1.19 & $\mathrm{C} 12 \mathrm{H} 22 \mathrm{O} 11$ & 342 & Lactose, \\
\hline 17 & 24.03 & 2.51 & $\mathrm{C} 9 \mathrm{H} 10 \mathrm{O} 2$ & 150 & 2-Methoxy-4-vinylphenol \\
\hline 18 & 24.167 & 0.25 & $\mathrm{C} 14 \mathrm{H} 30 \mathrm{O} 5 \mathrm{~S}$ & 310 & :d-Mannitol, 1-thiooctyl-1-deoxy \\
\hline 19 & 24.384 & 0.77 & C5H10O5 & 150 & Pentose \\
\hline 20 & 27.111 & 0.43 & $\mathrm{C} 18 \mathrm{H} 33 \mathrm{Cl} 3 \mathrm{O} 2$ & 386 & Trichloroacetic acid, hexadecyl ester \\
\hline 21 & 29.913 & 0.67 & C34H58O4 & 530 & Phthalic acid, ditridecyl ester \\
\hline 22 & 30.106 & 2.35 & $\mathrm{C} 16 \mathrm{H} 32 \mathrm{O} 2$ & 256 & n-Hexadecanoic acid \\
\hline 23 & 30.5 & 0.93 & $\mathrm{C} 24 \mathrm{H} 48 \mathrm{O} 2$ & 368 & Docosanoic acid, ethyl ester \\
\hline 24 & 32.094 & 1.31 & $\mathrm{C} 20 \mathrm{H} 40$ & 280 & 9-Eicosene, (E)- \\
\hline 25 & 32.193 & 0.13 & $\mathrm{C} 11 \mathrm{H} 20 \mathrm{O}$ & 168 & 6-Nonenal, 3,7-dimethyl- \\
\hline 26 & 33.12 & 1.09 & $\mathrm{C} 12 \mathrm{H} 22 \mathrm{O} 2$ & 198 & Acetic acid 9-decenyl ester \\
\hline
\end{tabular}


Alsaad \& Mohammed / Basrah J. Agric. Sci., 34(2), 204-219, 2021

\begin{tabular}{llllll}
\hline $\mathbf{2 7}$ & 33.248 & 13.54 & C14H26O & 210 & 7-Tetradecenal \\
\hline $\mathbf{2 8}$ & 33.447 & 0.38 & C10H18O & 154 & 5-Ethoxy-cyclooctene \\
\hline $\mathbf{2 9}$ & 33.82 & 0.97 & C14H28O & 212 & cis-11-Tetradecen-1-ol \\
\hline $\mathbf{3 0}$ & 33.855 & 0.79 & C8H13NO4 & 187 & $\begin{array}{l}\text { Acetic acid, 2-(2-buten-1-yl)-2-nitro-, } \\
\text { ethyl ester }\end{array}$ \\
\hline
\end{tabular}

Table (6): GC-MS analysis of EPeE of cactus $O$. dillenii (OD) fruit.

\begin{tabular}{|c|c|c|c|c|c|}
\hline $\mathrm{Pk}$ & $\mathrm{RT}(\min )$ & $\%$ Area & Formula & MW & Compounds name \\
\hline 1 & 3.077 & 6.57 & $\mathrm{C} 7 \mathrm{H} 14 \mathrm{O} 2$ & 130 & Acetic acid, pentyl ester \\
\hline 2 & 3.267 & 0.93 & $\mathrm{C} 2 \mathrm{H} 4 \mathrm{O} 2$ & 60 & Acetic acid \\
\hline 3 & 3.307 & 0.35 & $\mathrm{C} 2 \mathrm{H} 4 \mathrm{O} 2$ & 60 & Acetic acid \\
\hline 4 & 3.473 & 0.48 & C6H14O & 102 & Propane, 1-(1-methylethoxy)- \\
\hline 5 & 5.23 & 0.57 & $\mathrm{C} 9 \mathrm{H} 20 \mathrm{O} 2$ & 160 & Butane, 1,1-diethoxy-3-methyl- \\
\hline 6 & 18.912 & 11.39 & $\mathrm{C} 6 \mathrm{H} 12 \mathrm{O} 3$ & 132 & Butoxyacetic acid \\
\hline 7 & 21.459 & 1.3 & $\mathrm{C} 12 \mathrm{H} 14 \mathrm{O} 4$ & 222 & Phthalic acid, diethyl ester \\
\hline 8 & 21.538 & 0.59 & $\mathrm{C} 13 \mathrm{H} 26$ & 182 & 1-Tridecene \\
\hline 9 & 21.792 & 19.58 & C7H14O7 & 210 & d-Glycero-d-galacto-heptose \\
\hline 10 & 23.106 & 1.87 & C6H12O6 & 180 & Fructose \\
\hline 11 & 23.18 & 0.81 & C7H12O5 & 176 & $\begin{array}{l}\text { alpha.-D-Mannopyranoside, methyl 3,6- } \\
\text { anhydrous- }\end{array}$ \\
\hline 12 & 28.921 & 6.05 & C9H10O2 & 150 & 2-Methoxy-4-vinylphenol \\
\hline 13 & 28.995 & 7.38 & $\mathrm{C} 22 \mathrm{H} 42 \mathrm{O} 4$ & 370 & Hexanedioic acid, dioctyl ester \\
\hline 14 & 29.908 & 0.62 & C34H58O4 & 530 & Phthalic acid, ditridecyl ester \\
\hline 15 & 29.968 & 0.53 & $\mathrm{C} 13 \mathrm{H} 28$ & 184 & Nonane, 5- (1-methylpropyl \\
\hline 16 & 30.11 & 4.79 & $\mathrm{C} 21 \mathrm{H} 34 \mathrm{O}$ & 150 & 2-Methoxy-4-vinylphenol \\
\hline 17 & 30.227 & 0.29 & $\mathrm{C} 14 \mathrm{H} 26 \mathrm{O} 4$ & 258 & Oxalic acid, 6-ethyloct-3-yl ethyl ester \\
\hline 18 & 30.253 & 0.52 & $\mathrm{C} 10 \mathrm{H} 18$ & 138 & Naphthalene, decahedron \\
\hline 19 & 30.287 & 0.3 & $\mathrm{C} 8 \mathrm{H} 14 \mathrm{O} 2$ & 142 & 9-Oxabicyclo[4.2.1]nonan-2-ol \\
\hline 20 & 30.368 & 1.33 & $\mathrm{C} 21 \mathrm{H} 40 \mathrm{O} 5$ & 372 & Glycerylmonoricinoleate \\
\hline
\end{tabular}


Alsaad \& Mohammed / Basrah J. Agric. Sci., 34(2), 204-219, 2021

\begin{tabular}{llllll}
\hline $\mathbf{2 1}$ & 30.498 & 1.04 & $\mathrm{C} 24 \mathrm{H} 48 \mathrm{O} 2$ & 368 & Docosanoic acid, ethyl ester \\
\hline $\mathbf{2 2}$ & 30.633 & 0.76 & $\begin{array}{l}\mathrm{C} 22 \mathrm{H} 39 \mathrm{~F} 5 \mathrm{O} \\
2\end{array}$ & 430 & Nonadecylpentafluoropropionate \\
\hline $\mathbf{2 3}$ & 30.83 & 0.6 & $\mathrm{C} 10 \mathrm{H} 22$ & 142 & Nonane, 2-methyl \\
\hline $\mathbf{2 4}$ & 32.096 & 1.16 & $\mathrm{C} 20 \mathrm{H} 40$ & 280 & 3-Eicosene, (E)- \\
\hline $\mathbf{2 5}$ & 33.127 & 1.03 & $\mathrm{C} 13 \mathrm{H} 24$ & 180 & 6-Tridecane \\
\hline $\mathbf{2 6}$ & 33.257 & 22.08 & $\mathrm{C} 14 \mathrm{H} 26 \mathrm{O}$ & 210 & 7-Tetradecenal, (Z)- \\
\hline $\mathbf{2 7}$ & 33.52 & 0.43 & $\mathrm{C} 16 \mathrm{H} 30 \mathrm{O} 2$ & 254 & E-6-Tetradecen-1-ol acetate \\
\hline $\mathbf{2 8}$ & 33.547 & 0.58 & $\mathrm{C} 12 \mathrm{H} 20$ & 164 & Cyclohexane, 1,5-dimethyl-2,3-divinyl- \\
\hline $\mathbf{2 9}$ & 33.607 & 1.53 & $\mathrm{C} 23 \mathrm{H} 42 \mathrm{O} 2$ & 350 & Methyl 5,9-docosadienoate \\
\hline $\mathbf{3 0}$ & 33.851 & 4.54 & $\mathrm{C} 20 \mathrm{H} 40 \mathrm{O} 2$ & 312 & Eicosanoic acid \\
\hline
\end{tabular}

\section{Determination of (DPPH) free radical}

The scavenging of the DPPH assay depends on the decrease in absorbance as the DPPH radical received hydrogen radical or an electron from an antioxidant compound like phenols to become a stable molecule. This process can evaluate the ability of materials to donate electrons or hydrogen atoms to the reactive species (Paixao et al., 2007).

Results of free radical scavenging activity are given in table (7). Data in the figure indicated that DPPH free radical scavenging values increased proportionally and significantly to the sample concentration 20$80 \mu \mathrm{g} \cdot \mathrm{ml}^{-1}$. The highest scavenging activity was for EPeE, ranging from 34.8 to $90.3 \%$, followed by EPuE 32.9-89.9\%. All extracts were significantly different $(p<0.05)$, which means that free radicals react with the extracts considered effective electron donors, which completes the reaction by forming stable compounds. There are no significant differences $(p>0.05)$ between BHT and ethanolic extracts at a 60 and $80 \mu \mathrm{g} / \mathrm{ml}$ concentration. Extracts obtained using hexane (HPeE and HPuE) had significant differences, and the lowest scavenging activity ranged between 24.9-78.9 and 20.7-73.3\%, respectively. All extracts exhibited an appreciable scavenging effect, which could be because the extracts contain a high amount of antioxidant compounds, such as phenolic compounds and saturated fatty acid.

The scavenging activity of EPeE is in agreement with Faten \& Ali (2014), who found that the DPPH scavenging activity of $O$. ficus indica peels extracts by ethanol/water ranged from 62.72 to $90.4 \%$. Koubaa et al. (2015) studied three varieties of cactus. They showed that antioxidant activity by the DPPH method of $O$. undulata fruits was four times lower than that in $O$. ficus-indica and $O$. stricta fruits. In a study performed by Bakari et al. (2017), there is a positive relationship between the sample concentration and DPPH free radicals scavenging. Prior et al. (2005) 
showed that the ability to scavenge the DPPH

total phenols.

free radicals increased with a high content of

Table (7): DPPH scavenging activity of cactus $O$. dillenii (OD) extracts at different concentrations and comparative with Butylated Hydroxytoluene (BHT).

\begin{tabular}{|rccccc|}
\hline $\begin{array}{r}\text { Conc. } \\
\boldsymbol{\mu g . m l ^ { - 1 }}\end{array}$ & \multicolumn{4}{c}{ DPPH scavenging activity\% } \\
\cline { 2 - 6 } & $\mathbf{H P u E}$ & $\mathbf{H P e E}$ & $\mathbf{E P u E}$ & $\mathbf{E P e E}$ & $\mathbf{B H T}$ \\
\hline 20 & $20.7 \pm 0.05^{\mathrm{aA}}$ & $24.9 \pm 0.03^{\mathrm{aB}}$ & $32.9 \pm 0.02^{\mathrm{aC}}$ & $34.8 \pm 0.01^{\mathrm{aD}}$ & $38.5 \pm 0.06^{\mathrm{aE}}$ \\
\hline 40 & $32.0 \pm 0.02^{\mathrm{bA}}$ & $35.0 \pm 0.01^{\mathrm{bB}}$ & $57.6 \pm 0.08^{\mathrm{bC}}$ & $58.2 \pm 0.01^{\mathrm{bC}}$ & $67.6 \pm 0.01^{\mathrm{bD}}$ \\
\hline 60 & $56.0 \pm 0.01^{\mathrm{cA}}$ & $62.7 \pm 0.06^{\mathrm{cB}}$ & $71.8 \pm 0.06^{\mathrm{cC}}$ & $75.0 \pm 0.09^{\mathrm{cD}}$ & $81.1 \pm 0.08^{\mathrm{cD}}$ \\
\hline 80 & $73.3 \pm 0.15^{\mathrm{dA}}$ & $78.9 \pm 0.11^{\mathrm{dA}}$ & $89.9 \pm 0.02^{\mathrm{cB}}$ & $90.3 \pm 0.03^{\mathrm{dD}}$ & $93.0 \pm 0.07^{\mathrm{dD}}$ \\
\hline
\end{tabular}

The same small letters in the same row is not significantly different $(\mathrm{p} \leq 0.05)$, Mean with the same capital letters in the same column is not significantly different $(\mathrm{p} \leq 0.05)$ each value is the means \pm standard deviation $(n=3)$

\section{Conclusion}

The chemical composition of the Iraqi cactus (OD) was identified for the first time by the GC-MS technique. The moisture, acidity, TSS, and sugar values inpulp were higher than those in peels. The study confirms that pulp and peels of cactus pear fruit extracts are rich in natural antioxidants and are an important source of bioactive compounds, such as carbohydrate compounds, saturated fatty acids, and phenolic compounds, which may effectively scavenge the DPPH free radicals by ethanolic peels and pulp extracts in arang of $34.8-90.3 \%$ and $32.9-89.9 \%$ respectively. Hexane extracts scavenging ranges from 24.9-78.9\% and 20.7-73.3\%, respectively. These are good attributes to reduce the risk of several diseases due to their antioxidant activity.

\section{Acknowledgements}

We are thankful to Dr Asaad Y. Ayied, College of Agriculture, University of Basrah, who interpreted the data. We also thank Dr. Nadra Alsaad, Department of Computer Science, College for Pure Science Education, University of Basrah, who supports received this work by supplying us with the cactus fruits.

\section{References}

Aghajani, Z., Ali-Asghar, E., \& Akhbari M. (2015). Identification of volatile components and biological properties of the extracts of aerial parts of Pterocarya fraxinifolia L. Biological Forum - An International Journal, 7, 262-266. https://www.researchtrend.net/bfij/bf12/44\%20ZA HRA\%20AGHAJANI.pdf

Albano, C., Miceli, A., Negro, C., Tommasi, N., Gerardi, C., Mita G., Bellis, L.D., \& Blando., F. (2015). Betalains, phenols, and antioxidant capacity in cactus Pear [Opuntia ficus-indica (L.) Mill.] fruits from Apulia (South Italy) genotypes. Antioxidant, 4, 269-280. https://doi.org/10.3390/antiox4020269

Alsaad, A. J., Altemimi, A. B., Aziz,S. N., \& Lakhssassi, N. (2019). Extraction and identification of Cactus (Opuntia dillenii) seed oil and its added value for 
human health benefits. Pharmacogn Journal, 11, 19. https://doi.org/10.5530/PJ.2019.3

Anwar, M. M., \& Sallam, E. M. (2016). Utilisation of Prickly Pear Peels to Improve Quality of Pan Bread. Arab Journal of Nuclear Science and Applications, 49, 151-163. https://inis.iaea.org/search/search.aspx?orig_q=jour nal:\%22ISSN\%201110-0451\%22

AOAC (2010). Official Methods of Analysis of the Association of Official Analytical Chemists. 18th Ed. Published by the Association of Official Analytical Chemists. Arlington, Virginia. 2220pp. https://www.techstreet.com/standards/officialmethods-of-analysis-of-aoac-international-18thedition-revision-3?product_id=1678986

Bakari, S., Daoud, A., Felhi,S., Smaoui, S., Gharsallah, N., \& Kadri, A. (2017). Proximate analysis, mineral composition, phytochemical contents, antioxidant and antimicrobial activities and GC-MS investigation of various solvent extracts of cactus cladode. Food Science and Technology, 37, 286293. http://dx.doi.org/10.1590/1678-457X.20116

Bergaoui, A., Boughalleb, N., Ben Jannet, H., HarzallahShiric, F., El Mahjoub, M., \& Mighri, Z. (2007). Chemical composition and antifungal activity of volatiles from three Opuntia species growing in Tunisia. Pakistan Journal of Biological Sciences, $\quad 10, \quad 2485-2489$. http://dx.doi.org/10.3923/pjbs.2007.2485.2489

Bouzoubaâ, Z., Essoukrati, Y., Tahrouch, S., Hatimi, A., Gharby, S., \& Harhar, H. (2016). Phytochemical study of prickly pear from southern Morocco. Journal of Saudi Society of Agricultural Sciences, 15, 155-161. https://doi.org/10.1016/j.jssas.2014.09.002 (In Turkish)

Butera, D., Tesoriere, L., Di Gaudio, F., Bongiorno, A., Allegra, M., Pintaudi, A. M., Kohen, R., \& Livrea, M. A. (2002). Antioxidant activities of Sicilian prickly pears (Opuntia ficus-indica) fruit extracts and reducing properties of its betalains: betanin and indicaxanthin. Journal of Agricultural and Food Chemistry, 50, 6895-6901. https://doi.org/10.1021/jf025696p

Buyukokuroglu, M. E., Gulcin, I., Oktay, M., \& Kufrevioglu, O. I. (2001). In vitro antioxidant properties of dantrolene sodium). Pharmacological
Research, 44, 491-4. https://doi.org/10.1006/phrs.2001.0890

Duru, B., \& Turker, N. (2005). Changes in physical properties and chemical composition of cactus pear (Opuntia ficus-indica) Duringmaturation. Journal of the Professional Association for Cactus Development, 7, 22-33.

Faten, M. A. \& Ali, R. F. M. (2014). Antioxidant and anticancer activities of different constituents extracted from Egyptianprickly pear cactus (Opuntia ficus-Indica) peel. Biochemistry and Analytical Biochemistry, 3, 2-8. https://doi.org/10.4172/2161-1009.1000158

Fernández-López, J. A., Almela, L., Obón, J. M., \& Castellar, R. (2010). Determination of antioxidant constituents in cactus pear fruits. Plant Foods for Human Nutrition, 65, 253-259. https://doi.org/10.1007/s11130-010-0189-x

Flath, R. A. \& Takahashi, J. M. (1978). Volatile constituents of prickly Pear (Opuntia ficus indica Mill.(de CastillaVariety). Journal of Agricultural and Food Chemistry, 835-837. https://doi.org/10.1021/jf60218a053

Gulcin, I., Buyukokuroglu, M. E., Oktay, M., \& Kufrevioglu, O. I. (2003). Antioxidant and analgesic activities of turpentine of Pinus nigra Arn. subsp. pallsiana (Lamb.) Holmboe. Journal of Ethnopharmacology, 86, 51-58. https://doi.org/10.1016/s0378-8741(03)00036-9

Gurrieri, S., Miceli, L., Lanza, C. M., Tomaselli, F., Bonomo, R. P., \& Rizzarelli, E. (2000). Chemical scharacterisation of Sicilian prickly pear (Opuntia ficus indica) and perspectives for the storage of its juice. Journal of Agricultural and Food Chemistry, 48, 5424-5431. https://doi.org/10.1021/jf9907844

Jacobo, M. C. (2001). Cactus pear breeding and domestication. Plant Breeding Reviews, 20, 135166. https://doi.org/10.1002/9780470650189.ch5

Jaiswal, D., Rai, P. K., \& Watal, G. (2009). Antidiabetic effect of Withania coagulants in the experimental rat. Indian Journal of Clinical Biochemistry, 24, 8893.https://doi.org/10.1007/s12291-009-0015-0

Koubaa, M., Ktata, A., Bouaziz, F., Driss, D., Ghorbel, R. E. \& Chaabouni, S. E. (2015). Solvent extract from Opuntia stricta fruit peels Chemical composition and biological activities. Free Radicals 


\section{Alsaad \& Mohammed / Basrah J. Agric. Sci., 34(2), 204-219, 2021}

\& Antioxidants, 5, 5259.https://doi.org/10.5530/fra.2015.2.2

Madrigal-Santillán, E., García-Melo, F., MoralesGonzález, J. A., Vázquez-Alvarado, P., MuñozJuárez, S., Zuñiga-Pérez, C., Sumaya-Martínez, M. T., Madrigal-Buiaidar, E., \& Hernández-Ceruelos, A. (2013). The antioxidant and anticlastogenic capacity of prickly pear juice. Nutrients, 5, 41454158. https://doi.org/10.3390/nu5104145

Makris, D. P., Boskou, G., \& Andrikopoulos, N. K. (2007). Polyphenolic content and in vitro antioxidant characteristics of the wine industry and other agri-food solid waste extracts, Journal of Food Composition and Analysis, 20, 125-132. https://doi.org/10.1016/j.jfca.2006.04.010

Matias, A., Nunes, S. L., Poejo, J., Mecha, E., Serra, A. T., Amorim Madeira, P. J., Bronze, M. R., \& Duarte, C. M. M. (2014). Antioxidant and antiinflammatory activity of a flavonoid-rich concentrate recovered from Opuntia ficus-indica juice. Food \& Function, 5, 3269-3280. https://doi.org/10.1039/c4fo00071d

Moßhammer, M. R., Stintzing, F. C., \& Carle, R. (2006). Cactus Pear fruits (Opuntia spp.): A review of processing technologies and current uses. Journal of the Professional Association for Cactus Development, $\quad 8, \quad 1-25$. https://www.researchgate.net/publication/27965290 3

Morales, J., \& 'Enez-Perez, S. J. (2001). Free radical scavenging capacity of Maillard reaction products as related to colour and fluorescence. Food Chemistry, 72, 119-125. https://doi.org/10.1016/S0308-8146(00)00239-9

Osorio-Esquivel, O., Ortiz-Moreno, A., Álvarez, V. B., Dorantes-Álvarez, L., \& Giusti, M. M. (2011). Phenolics, betacyanins) and antioxidant activity in Opuntia joconostle fruits. Food Research International, $\quad 44, \quad 2160 \quad-2168$. https://doi.org/10.1016/j.foodres.2011.02.011

Paixao, N., Perestrelo, R., Marques, J. C., \& Camara, J. S. (2007). Relationship between antioxidant capacity and total phenolic content of red rose and white wines. Food Chemistry, 105, 204-214. https://doi.org/10.1016/j.foodchem.2007.04.017

Parish, J., \& Felker, P. (1997). Fruit quality and production of Cactus pear (Opuntia spp.). Fruit clones selected for increased frost hardiness, Journal of Arid Environments, 37, 123-143. https://doi.org/10.1006/jare.1997.0261

Piga, A. (2004). Cactus pear: A fruit of nutraceutical and functional importance. Journal Professional Association for Cactus Development, 6, 9-22.

Prior, R. L., Wu, X., \& Schaich, K. (2005). Standardised methods for the determination of antioxidant capacity and phenolics in foods and dietary supplements. Journal of Agricultural and Food Chemistry, 53, 4290-4302. https://doi.org/10.1021/jf0502698

Rajeswari, G., Murugan, M., \& Mohan, V. R. (2012). GC-MS analysis of bioactive components of Hugonia mystax L. (Linaceae). Research Journal of Pharmaceutical, Biological, and Chemical Sciences, 3, 301-318.

Ramadan, M. F., \& Mörsel, J. T. (2003). Oil Cactus pear (Opuntia ficus-indica L.). Food Chemistry, 82, 339-345. https://doi.org/10.1016/S03088146(02)00550-2

Sáenz, C. (2000). Processing technologies: an alternative for Cactus pear (Opuntia spp.) fruits and cladodes. Journal of Arid Environments, 46, 209-225. https://doi.org/10.1006/jare.2000.0676.

(In Turkish).

Sarkadei, S., \& Howell, N. K. (2007). Effect of natural antioxidants on stored freeze-dried food product formulated using horse mackerel (Trachurus trachurus). Journal of Science and Technology, 43, 309-315. https://doi.org/10.1111/j.13652621.2006.01435.x

Shahidi, F., Janitha, P. K., \& Wanasundara, P. D. (1992). Phenolic antioxidants. Critical Reviews in Food Science and Nutrition, 32, 67-103. https://doi.org/10.1080/10408399209527581

Sharma, C., Rani, S., Kumar, B., Kumar, A., \& Raj, V. (2015). Plant Opuntia dillenii: A review on its traditional uses, phytochemical, and pharmacological properties. EC Pharmaceutical Science, $\quad 11, \quad 29-43$. https://www.semanticscholar.org/paper/PlantOpuntia-dillenii\%3A-A-Review-on-It\%27sUses\%2C-and-SharmaRani/e7cdd1e6ac4dc6ef2e6b4987d84e39327eb6bac 9 
Valente, L. M. M., daPaixão, D., do Nascimento, A. C., dos Santos, P. F. P., Scheinvar, L. A., Moura, M. R. L., Tinoco, L. W., Gomes, L. N. F., \& da Silva, J. F. M. (2010). Antiradical activity, nutritional potential, and flavonoids of the cladodes of Opuntia monacantha (Cactaceae). Food Chemistry, 123, 1127-1131.

https://doi.org/10.1016/j.foodchem.2010.05.074

Yadav, L., \& Chakravarty, A. (2013). Effect of keeping time period on acidityof fruit juices and determination of fungal growth in fruit juices. Asian Journal of Home Science, 8, 166-169. https://www.semanticscholar.org/paper/Effect-ofKeeping-Time-Period-on-Acidity-of-Fruit-Yadav-
Chakravarty/89a25fb7beb9a2505ef226ab0b2082ade9 e6cd66

Zhang, W., Li, B., Han, L., \& Zhang, H. (2009). Antioxidant activities of extracts from (Areca catechu L.) flower husk and seed. African Journal of Biotechnology, 8, 3887-3892. https://www.ajol.info/index.php/ajb/index

Zito, P., Sajeva, M., Bruno, M., Rosselli, S., Maggio, A., \& Senatore, F. (2013). Essential oils composition of two Sicilian cultivars of Opuntia ficus-indica (L.) Mill (Cactaceae) fruits (prickly pear). Natural Product Research, 27, 1305- 1314. https://doi.org/10.1080/14786419.2012.734823.

دراسة الفعالية المضادة للاكسدة لفاكهة الصبار Opuntia dellienii (اللب والقشور) وتشخيص مركباته GC-MS الفعالة بواسطة

\author{
عاليه جميل علي السعد* و لينا سمير محمد \\ قسم علوم الاغذية ,كلية الزر اعة,جامعة البصرة , العراق
}

المستخلص: هدفت الدراسة الحالية إلى تحديد وتثخيص المركبات الكيميائية الموجودة في مستخلصات فاكهة الصبار Opuntia dillenii (OD) الفاكهة (اللب والقشور). حملت مستخلصات لب الفاكهة وقثورها الرموز : مستخلص لب الهكسان (HPuE) ، مستخلص قشور الهكسان (HPeE) ، مستخلص اللب الإيثانولي (EPuE) ومستخلص القشور الإيثانولي (EPeE). فحصت المركبات الفعالة بواسطة مقياس الطيف الكتلي اللوني للغاز (HC-MS). احتوى HPuE و HPeE ملى 40 مركبًا حيويًا وتم اكتثاف 60 مركبًا حيويًا في EPuE و EPeE. سجل نشاط اقتناص الجذور الحرة DPPH مستوى عالٍ من التثبيط تراوح من

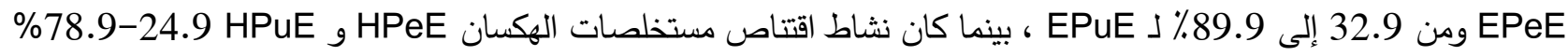
و 20.7-73.3٪ على التوالي بتراكيز تراوحت بين 20-80 ميكروغرام مل-1. حظي هذا النشاط باهتمام كبير بسبب وظائفه

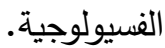
الكلمات المفتاحية: الصبار ، طريقة DPPH ، فواكه ، GC-MS ، القياسات الفيزيوكيميائية. 